\title{
20 I7 Validation of electrocardiographic and biochemical estimates of first acute myocardial infarct size using cardiac magnetic resonance imaging
}

\author{
Thomas N Martin*, Galen Wagner, Allan Pettigrew, Bjoern Groenning, \\ Robin Weir, Charles Maynard, Andrew Flapan and Henry Dargie
}

Address: Glasgow University, Glasgow, UK

* Corresponding author

from I th Annual SCMR Scientific Sessions

Los Angeles, CA, USA. I-3 February 2008

Published: 22 October 2008

Journal of Cardiovascular Magnetic Resonance 2008, I0(Suppl I):A286 doi:I0.I I86/I532-429X-I0-SI-A286

This abstract is available from: http://jcmr-online.com/content/I0/SI/A286

(c) 2008 Martin et al; licensee BioMed Central Ltd.

\section{Introduction}

The extent of damage following acute myocardial infarction is closely related to a prognosis so it is of clinical importance to make an accurate in vivo estimate of infarct size. Routinely available data includes cardiac biomarkers and the 12 lead ECG.

\section{Purpose}

This study investigates the relationship between Troponin I (TnI), the ECG derived Selvester score (SS) and contrast enhanced Magnetic Resonance Imaging (ceMRI) measures of infarct size.

\section{Methods}

80 consecutive patients with first acute coronary syndrome underwent ceMRI at a mean (SD) of 64 (23) hours from chest pain on a Siemens Sonata $1.5 \mathrm{~T}$ system using a phased array chest coil. Left ventricular ejection fraction (LVEF) was evaluated using the steady state free precession breath-hold sequence. CeMRI was performed 15 minutes after peripheral injection of $0.2 \mathrm{mmol} / \mathrm{kg}$ gadolinium-DTPA using a breath-hold segmented gradientecho inversion-recovery sequence. The scans were assessed by 2 experienced observers and the area of delayed hyperenhancement (DE) was planimetered manually. 23 patients were excluded based on ceMRI findings: 11 had DE in > 1 coronary territory; 3 had subendocardial sparing pattern of DE; and 9 were TnI positive with no evidence of DE. 57 patients (43 male) of mean (SD) age 59.8
(12.5) years were included in final analysis. Serum TnI was measured 8-12 hours following onset of chest pain. The complete 50-criteria, 31 point Selvester QRS scoring system was performed at the time of initial ceMRI by the Duke ECG core lab.

\section{Results}

Admission LVEF significantly correlates with DE $(\mathrm{r}=$ $0.45,0.001)$ and SS $(\mathrm{r}=-0.52, \mathrm{p}<0.001)$ but not TnI. Infarct size by ceMRI is moderately correlated with TnI $(\mathrm{r}=$ $0.69, \mathrm{p}<0.005)$ and SS $(\mathrm{r}=0.59, \mathrm{p}<0.005)$. The $\mathrm{R}^{2}$ value for SS alone is 0.41 and rises to 0.72 when combined with TnI. The Bland Altman limits of agreement are wide for both SS $($ mean difference $=15($ range $-11,41))$ and TnI (mean difference $=-18$ (range $-98,62)$ ).

\section{Conclusion}

Both TnI and SS correlate with acute infarct size by ceMRI and this correlation improves when they are used in combination. However the limits of agreement are wide, suggesting that a similar process is being measured but that they should not be used interchangeably. 